\title{
Specific IgE for Fag e 3 Predicts Oral Buckwheat Food Challenge Test Results and Anaphylaxis: A Pilot Study
}

\author{
Noriyuki Yanagida ${ }^{a}$ Sakura Sato $^{\mathrm{b}}$ Nobuyuki Maruyama ${ }^{c}$ Kyohei Takahashi ${ }^{\mathrm{a}}$ \\ Ken-ichi Nagakura ${ }^{a}$ Kiyotake Ogura ${ }^{a}$ Tomoyuki Asaumia ${ }^{a}$ Motohiro Ebisawab \\ ${ }^{a}$ Department of Pediatrics, Sagamihara National Hospital, Kanagawa, Japan; bepartment of Allergy, Clinical \\ Research Center for Allergy and Rheumatology, Sagamihara National Hospital, Kanagawa, Japan; ' Laboratory of \\ Food Quality Design and Development, Graduate School of Agriculture, Kyoto University, Kyoto, Japan
}

\section{Keywords}

Buckwheat · Food allergy · Food hypersensitivity · Oral food challenge $\cdot$ Immunoglobulin $\mathrm{E}$

\section{Abstract \\ Background: Buckwheat (BW) is the source of a life-threat- ening allergen. Fag e 3-specific serum IgE (slgE) is more use- ful than BW-slgE for diagnosis; however, it is unknown whether Fag e 3-slgE can predict oral food challenge (OFC) results and anaphylaxis. This study aimed to clarify the effi- cacy of Fag e 3-slgE in predicting OFC results and anaphy- laxis. Methods: We conducted a retrospective review of BW- and Fag e 3-slgE data obtained using the ImmunoCAP ${ }^{\circledR}$ as- say system and fluorescent enzyme-linked immunosorbent assay from children who underwent OFC using 3,072 mg of BW protein between July 2006 and March 2014 at Sagami- hara National Hospital, Kanagawa, Japan. Results: We ana- lyzed 60 patients aged 1.9-13.4 years (median 6.0 years); 20 (33\%) showed objective symptoms upon BW OFC. The pa- tients without symptoms had significantly lower Fag e 3-slgE than those with non-anaphylactic $(p<0.001)$ and anaphylac- tic reactions to $\mathrm{BW}(p=0.004)$. Fag e 3-slgE was the only test-}

ed factor that significantly predicted positive OFC results (odds ratio 8.93, 95\% confidence interval 3.10-25.73, $p<$ $0.001)$ and OFC-induced anaphylaxis $(2.67,1.12-6.35, p=$ 0.027). We suggest that a threshold Fag e 3-slgE level of 18.0 $\mathrm{kU}_{\mathrm{E}} / \mathrm{L}$ has $95 \%$ probability of provoking a positive reaction to BW. Conclusions: Fag e 3-slgE predicted OFC results and OFC-induced anaphylaxis. We further emphasize paying careful attention to the risk of BW OFC-induced anaphylaxis.

\section{Introduction}

Buckwheat (BW) has been widely consumed in Asian countries and is increasing in popularity in the USA and Europe $[1,2]$. However, it can be a potent source of allergens and can cause life-threatening anaphylaxis. In Japan, BW is the fourth-most common cause of food-induced anaphylaxis [3-5]. The estimated prevalence of BW allergy in school-children in Japan is $0.22 \%$ [6]. More than

Edited by: R. van Ree, Amsterdam.

\section{KARGER}

E-Mail karger@karger.com www.karger.com/iaa (c) 2018 The Author(s)

Published by S. Karger AG, Basel

Karger

Open access

This article is licensed under the Creative Commons AttributionNonCommercial-NoDerivatives 4.0 International License (CC BYNC-ND) (http://www.karger.com/Services/OpenAccessLicense). Usage and distribution for commercial purposes as well as any distribution of modified material requires written permission.
Correspondence to: Dr. Noriyuki Yanagida

Department of Pediatrics, Sagamihara National Hospital

18-1, Sakuradai, Minami-ku

Sagamihara-City, Kanagawa 252-0392 (Japan)

E-Mail n-yanagida@ sagamihara-hosp.gr.jp 
Fig. 1. Study participation.

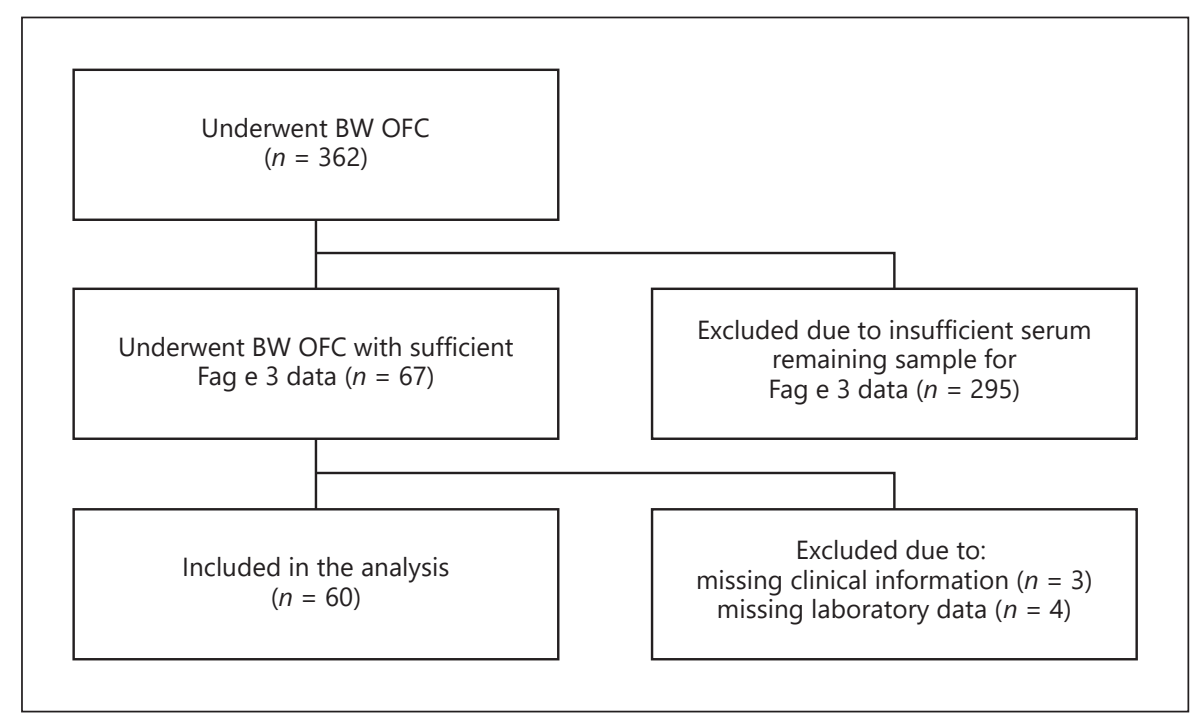

half of challenge-positive patients for BW present anaphylaxis [4]. BW serum-specific IgE (sIgE) levels have been useful for diagnosing BW allergy [4]; however, Fag e 3 (7S globulin, vicilin fragment) is a novel candidate as a more useful BW allergen component for diagnosis [7] than BW alone. Currently, the most accurate diagnosis method for BW allergy is oral food challenge (OFC). Our study aimed to clarify the efficacy of Fag e 3-sIgE to predict OFC results.

\section{Materials and Methods}

\section{Study Population and Enrollment}

We retrospectively analyzed data from children who underwent an OFC with BW in Sagamihara National Hospital, Kanagawa, Japan, between July 2006 and April 2014. About 60\% of patients were referred from primary care clinics, and the rest were admitted directly without referral. Patients who were allergic or suspected to be allergic to BW were diagnosed based on the presence of a history of immediate reaction to BW or past or current atopic dermatitis with positive $\mathrm{BW}$-sIgE. We enrolled only those undergoing their first BW OFC and did not have missing clinical or laboratory data.

\section{Oral Food Challenge}

In total, $64 \mathrm{~g}$ of BW noodles containing 3,072 $\mathrm{mg}$ of BW protein were used for OFC as previously described [4] (based on the 2017 Japanese Pediatric Guidelines for Food Allergy [8]). Treatments for provoked reaction were based on the European Academy of Allergy and Clinical Immunology's food allergy and anaphylaxis guidelines [9]. Anaphylaxis was defined according to World Allergy Organization Anaphylaxis Guidelines [10], and severity of symptoms was defined according to Japanese anaphylaxis guidelines $[8,11]$.

Fag e 3 for Buckwheat Allergy

\section{Laboratory Data}

We assessed BW-sIgE within 180 days of the OFC using the ImmunoCAP ${ }^{\circledR}$ assay system (Thermo Fisher Scientific/Phadia, Uppsala, Sweden). Fag e 3-sIgE was analyzed using stored serum samples via a fluorescent enzyme-linked immunosorbent assay (ELISA) at Kyoto University using previously described methods [7]. The serum sample was stored at $-80^{\circ} \mathrm{C}$ for a median of 2.5 years (range $0.5-8.2$ years) before analysis. These stored conditions did not affect the stability of sIgE [12-14].

\section{Statistical Analysis}

We expressed data as median values and ranges, used the Mann-Whitney U or the Fisher exact test for statistical comparisons, as appropriate, and considered $p$ values of $<0.05$ as statistically significant. We performed univariate and multivariate analyses using logistic regression. To create probability curves, we used regression analysis after logarithmic transformation of sIgE values, as previously published [15]. We used SPSS v24.0 (IBM Corp., Armonk, NY, USA) to perform all statistical analyses.

\section{Ethical Considerations}

In accordance with the Declaration of Helsinki, the study design and risks of anaphylaxis during the OFC were fully explained to patients and their guardians, both orally and in writing, and written informed consent was obtained from all participants for the OFC and publication of the data. All patient data were anonymized prior to analysis. We obtained approval from the institutional review board of Sagamihara National Hospital.

\section{Results}

\section{Patient Background}

In total, 362 patients underwent an OFC (Fig. 1). We excluded 295 patients owing to insufficient serum sam- 
Table 1. Clinical and demographic characteristics of patients

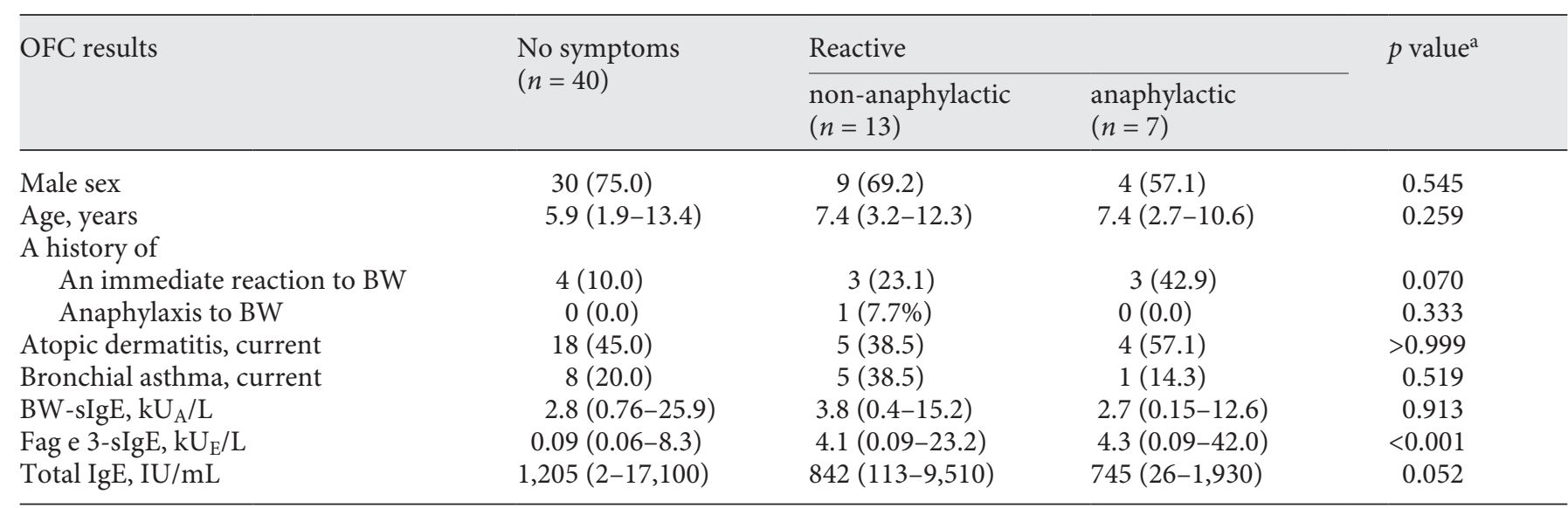

Values are expressed as $n(\%)$ or median (range). BW, buckwheat; sIgE, specific immunoglobulin E.

${ }^{a}$ Represents the statistical significance between negative cases and reactive cases. No significant difference among the 3 groups (no symptoms, non-anaphylactic, and anaphylactic) was seen without Fag e $3 \operatorname{sigE}(p<0.001)$.

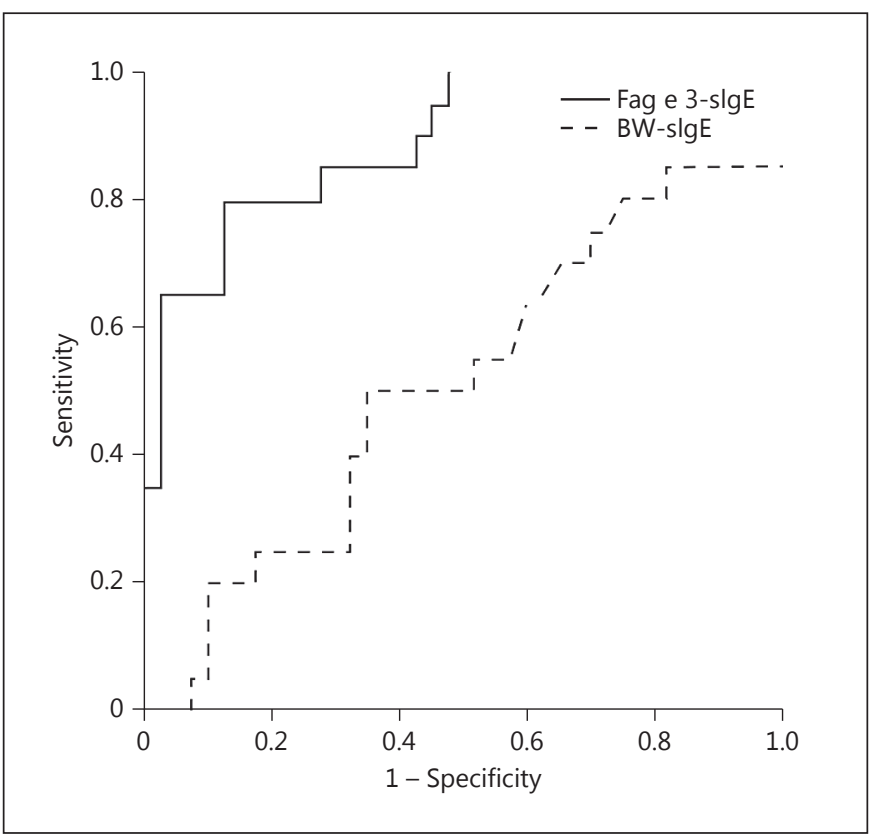

Fig. 2. ROC curve analysis to diagnose buckwheat allergy. BW, buckwheat; ROC, receiver-operating characteristic; sIgE, specific IgE.

ples for sIgE tests, 3 owing to a lack of clinical information, and 4 owing to missing laboratory data. The remaining 60 patients with suspected or definitive BW allergies were 1.9-13.4 years of age (median 6.0 years; Table 1), 10 of whom had a history of reactions to BW, and 1 with a
Table 2. Diagnostic performance of BW- and Fag e 3-sIgE

\begin{tabular}{lll}
\hline & BW-sIgE & Fag e 3-sIgE \\
\hline AUC & 0.509 & 0.893 \\
95\% CI & $0.348-0.669$ & $0.808-0.977$ \\
Optimal cut-off value, $\mathrm{kU}_{\mathrm{E}} / \mathrm{L}$ & 3.8 & 0.3 \\
Sensitivity, \% & 50.0 & 80.0 \\
Specificity, \% & 65.0 & 87.3
\end{tabular}

AUC, area under the curve; BW, buckwheat; CI, confidence interval; sIgE, specific immunoglobulin E.

history of anaphylaxis due to BW. Median BW- and Fag e 3 -sIgE levels were $2.8 \mathrm{kU}_{\mathrm{A}} / \mathrm{L}$ (range $0.15-25.9$ ) and 0.14 $\mathrm{kU}_{\mathrm{E}} / \mathrm{L}$ (range $0.06-42.0$ ), respectively.

\section{OFC Results}

Twenty patients (33\%) reacted, all with objective symptoms. Skin reactions were most common (15 [75\%]), followed by respiratory $(12[60 \%])$, gastrointestinal (11 [55\%]), neurological (2 [10\%]), and cardiovascular (1 [5\%]) symptoms. We observed anaphylactic reactions in 7 patients (35\%). Moderate and severe symptoms, as defined in Japanese Guidelines for Food Allergy [8], were present in $9(45 \%)$ and 5 patients $(25 \%)$, respectively.

Fourteen patients $(70 \%)$ received treatment. Oral antihistamine was the most frequent treatment, adminis-
10

Int Arch Allergy Immunol 2018;176:8-14 DOI: $10.1159 / 000487135$
Yanagida/Sato/Maruyama/Takahashi/ Nagakura/Ogura/Asaumi/Ebisawa 
Table 3. Univariate and multivariate analyses of factors related to positive OFC results

\begin{tabular}{|c|c|c|c|c|}
\hline $\begin{array}{l}\text { Risk factors for positive OFC results } \\
(n=60)\end{array}$ & $\begin{array}{l}\text { Crude OR } \\
(95 \% \mathrm{CI})\end{array}$ & $p$ value & $\begin{array}{l}\text { Adjusted } \mathrm{OR}^{\mathrm{a}} \\
(95 \% \mathrm{CI})\end{array}$ & $p$ value \\
\hline BW-sIgE (10-fold increments) & $0.800(0.223-2.878)$ & 0.733 & $1.026(0.269-3.924)$ & 0.970 \\
\hline Fag e 3 -sIgE (10-fold increments) & $8.930(3.099-25.729)$ & $<0.001$ & $8.933(2.927-27.265)$ & $<0.001$ \\
\hline History of an immediate reaction to BW & $3.857(0.944-15.763)$ & 0.060 & - & \\
\hline
\end{tabular}

BW, buckwheat; sIgE, specific immunoglobulin E; CI, confidence interval; OFC, oral food challenge; OR, odds ratio.

a Adjusted according to a history of an immediate reaction to BW.

Table 4. Univariate and multivariate analyses of factors related to OFC-induced anaphylaxis

\begin{tabular}{|c|c|c|c|c|}
\hline $\begin{array}{l}\text { Risk factors for OFC-induced } \\
\text { anaphylaxis }(n=60)\end{array}$ & $\begin{array}{l}\text { Crude OR } \\
(95 \% \mathrm{CI})\end{array}$ & $p$ value & $\begin{array}{l}\text { Adjusted OR } \\
(95 \% \mathrm{CI})\end{array}$ & $p$ value \\
\hline BW-sIgE (10-fold increments) & $0.62(0.096-4.02)$ & 0.617 & $0.89(0.13-6.09)$ & 0.903 \\
\hline Fag e 3-sIgE (10-fold increments) & $2.67(1.12-6.35)$ & 0.027 & $2.33(0.96-5.66)$ & 0.061 \\
\hline History of an immediate reaction to BW & $4.93(0.91-26.85)$ & 0.065 & - & \\
\hline
\end{tabular}

BW, buckwheat; sIgE, specific immunoglobulin E; CI, confidence interval; OFC, oral food challenge; OR, odds ratio.

a Adjusted according to a history of an immediate reaction to BW.

tered in 13 patients $(65 \%) . \beta_{2}$ stimulant inhalation was administered to 11 patients (55\%), intravenous steroids to $6(30 \%)$, and intramuscular adrenaline injections to 2 (10\%). Of 7 patients who showed anaphylaxis, 5 did not need adrenaline injections because of a rapid improvement in the respiratory symptoms following $\beta_{2}$ stimulant inhalation $(n=4)$, or improvement in moderate skin symptoms and repeated vomiting after the administration of intravenous antihistamines and steroids.

\section{Diagnostic Performance of BW and Fag e 3-sIgE}

The diagnostic performance of BW- and Fag e 3-sIgE was compared by calculating their respective area under the curve (AUC) and optimal cut-off values. The AUC for BW and Fag e 3-sIgE was 0.509 and 0.893 , respectively (Fig. 2; Table 2). The optimal cut-off values were 3.8 $\mathrm{kU}_{\mathrm{A}} / \mathrm{L}$ and $0.3 \mathrm{kU}_{\mathrm{E}} / \mathrm{L}$, respectively.

\section{Risk Factors for Positive Challenge}

Patients with positive OFC results more frequently had higher Fag e 3-sIgE (10-fold increments; odds ratio [OR] 8.93, 95\% confidence interval [CI] 3.10-25.73, $p<$ 0.001 ) (Table 3 ). There was no significant difference be- tween BW-sIgE and positive OFC results. There was also no significant difference between a history of an immediate reaction to $\mathrm{BW}$ and positive OFC. In a multivariate analysis adjusted for the presence of immediate reactions [4], Fag e 3-sIgE (10-fold increments, adjusted OR 8.93, 95\% CI 2.93-27.27, $p<0.001$ ) remained a significant factor for positive OFC results. In a univariate analysis, Fag e 3-sIgE was the only significant factor for OFC-induced anaphylaxis (10-fold increments, OR 2.67, 95\% CI 1.12$6.35, p=0.027$ ) (Table 4), although this was nonsignificant in the multivariate analysis adjusted for immediate reaction.

\section{Fag e 3-sIgE and Symptoms during OFC}

Patients without symptoms had significantly lower Fag e 3-sIgE than those with non-anaphylactic $(p<0.001)$ or anaphylactic symptoms to BW $(p=0.004)$ (Fig. 3). Among challenge-positive patients $(n=20)$, those with gastrointestinal symptoms had higher Fag e 3-sIgE levels than those without such symptoms $(p=0.038)$. Although similar trends were observed for skin reactions and anaphylaxis with the Fag e 3-sIgE concentration, these were not statistically significant. 


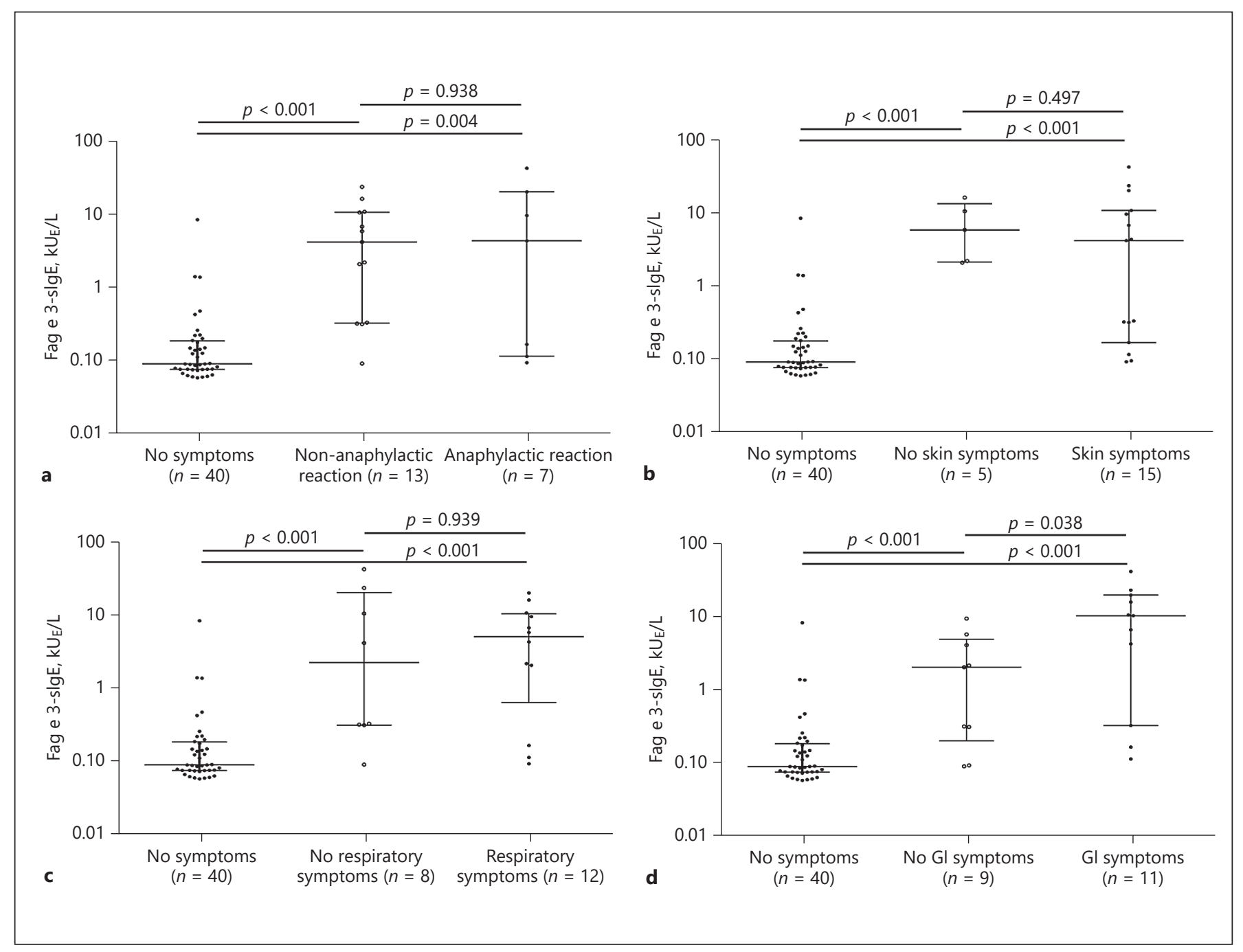

Fig. 3. Fag e 3-sIgE and symptoms during oral food challenge (OFC). a Fag e 3-sIgE among patients with no symptoms, nonanaphylactic reactions, and anaphylactic reactions during OFC. b-d Fag e 3-sIgE among patients with no symptoms; patients showing symptoms other than skin, respiratory, and gastrointestinal (GI) symptoms; and patients with skin, respiratory, and GI symptoms, respectively, during OFC. Median and interquartile range are indicated by horizontal lines.

\section{Probability of Failed Challenge and Anaphylaxis during OFC}

Figure 4 presents fitted predicted probability curves for positive challenge and OFC-induced anaphylaxis at a given Fag e 3-sIgE concentration. We suggest that a threshold Fag e 3 -sIgE concentration of $18.0 \mathrm{kU}_{\mathrm{E}} / \mathrm{L}$ would have an approximately $95 \%$ probability of inducing any reaction to $\mathrm{BW}$ (Table 5). We were unable to calculate the sIgE concentration level at which approximately $95 \%$ of the patients are predicted to experience anaphylaxis (the $95 \%$ predictive decision point). The $50 \%$ predictive decision point for Fag e 3-sIgE to anaphylaxis was $75.4 \mathrm{kU}_{\mathrm{E}} / \mathrm{L}$.

\section{Discussion}

This was the first study to calculate the $95 \%$ predictive decision point for Fag e 3-sIgE. Although the usefulness of Fag e 3-sIgE has been reported, these studies included patients with a convincing history [6], whereas we included only OFC-proven BW-allergic and nonallergic patients. Consistent with a previous report [4], we observed frequent anaphylactic reactions and severe symptoms. Furthermore, we were able to estimate the risk of OFCinduced anaphylaxis, which clinicians should be aware of during OFC.
Yanagida/Sato/Maruyama/Takahashi/ Nagakura/Ogura/Asaumi/Ebisawa 
Table 5. Clinical efficacy of Fag e 3-sIgE for predicting a positive oral food challenge to buckwheat

\begin{tabular}{llll}
\hline Probability & 50\% pred. probability & $90 \%$ pred. probability & 95\% pred. probability \\
\hline Fag e 3-sIgE, $\mathrm{kU}$ E $/ \mathrm{L}$ & 0.8 & 8.2 & 18.0 \\
$95 \%$ CI & 0.4 to 3.0 & 2.4 to 200.0 & -4.3 to 871.0 \\
\hline
\end{tabular}

CI, confidential interval; sIgE, specific immunoglobulin E; pred., predicted.

BW OFC may induce severe reactions [4]. Therefore, knowing the probability for a reaction and anaphylaxis may help assess the risk of a positive OFC result and OFCinduced anaphylaxis prior to the challenge. Generally, many patients need BW OFC because BW-sIgE can only roughly predict $\mathrm{OFC}$ results. However, similar to a previous study [6], Fag e 3-sIgE was more useful than BW-sIgE for predicting positive reactions. It was also useful for predicting OFC results. This suggests that OFC with BW in patients with Fag e 3 -sIgE $>18 \mathrm{kU}_{\mathrm{E}} / \mathrm{L}$ should be avoided. These results were derived using ELISA. Probability curves of different assays cannot be applied interchangeably [16]; therefore, it is unknown whether other methods would achieve $95 \%$ predictive decision points similar to those obtained for Fag e 3-sIgE.

In some foods, including those with minor allergens, 95\% predictive decision points were reported $[17,18]$. Component-resolved diagnosis (CRD) improves the accuracy of diagnosing IgE-mediated food allergy [19]. In some allergenic foods, like wheat and peanut, CRD ( $\omega-5$ gliadin, Ara h 2) provided a more precise diagnostic performance than crude food allergen-sIgE does, and only CRD showed $95 \%$ predictive decision points [20,21]. Although BW-sIgE is useful [4], the $95 \%$ predictive decision points were not calculated. Our study also revealed that Fag 3e 3, a component of BW, is more useful than crude BW.

There were some limitations to our study. First, the OFCs were not double-blinded, placebo-controlled challenges. However, all OFC-positive patients had objective symptoms, including anaphylaxis; therefore, this should not actually affect the conclusions. Second, we performed a skin prick test (SPT) for only a few patients; combining the SPT and Fag e 3-sIgE may improve diagnostic performance. Moreover, we could not compare Fag e 3-sIgE and the SPT. Fag e 3-slgE is currently commercially unavailable, and the SPT test is cheaper and faster. Accordingly, there is a need for further study to compare the two. Currently, the commercial availability of Fag e 3-sIgE is limited; therefore, its use in clinical settings is also limited.

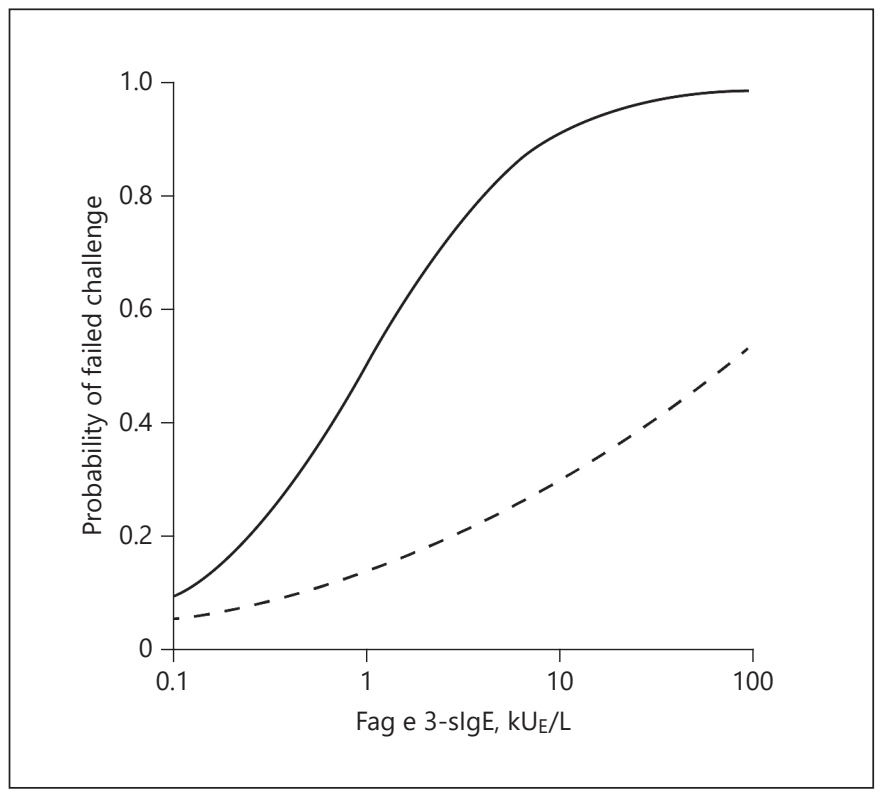

Fig. 4. Probability curves representing positive outcomes of oral food challenge (OFC) and anaphylaxis during OFC at a given Fag e 3-sIgE in 60 patients. The solid line represents the probability of an objective reaction $(n=20)$. The dotted line represents the probability of an anaphylactic reaction $(n=7)$.

Finally, there was a potential selection bias in our study. For example, patients with a strong history of anaphylaxis might avoid OFC. We excluded many patients with missing Fag e 3-sIgE data and included only 60 with BW OFC, primarily because many patients had an insufficient residual serum sample. The differences in Fag e 3-sIgE concentrations between patients with and without gastrointestinal symptoms, but not between those with other symptoms and anaphylaxis, may have originated from the small sample size. Larger prospective studies are needed.

In conclusion, Fag e 3-sIgE predicted OFC results and OFC-induced anaphylaxis. We further emphasize paying careful attention to the risk of BW OFC-induced anaphylaxis. 


\section{Acknowledgements}

All aspects of this study, including study design, data collection, analysis, and interpretation of data, were supported by the Health and Labour Sciences Grant for Research into Allergic Disease and Immunology from the Ministry of Health, Labour, and Welfare, Japan, (M.E.; grant No. 201414009A). The study was also partially supported by the Practical Research Project for Allergic Disease and Immunology from the Japan Agency for Medical Research and Development, AMED (15ek0410019h0101) for the publica- tion of the paper. We are particularly grateful to all the pediatricians, nutritionists, and nurses who participated in patient recruitment and data collection at Sagamihara National Hospital.

\section{Disclosure Statement}

M. Ebisawa is on the DBV Technologies Scientific Advisory Board, and received lecture fees from Pfizer and Siemens. The remaining authors declare no relevant conflicts of interest.

\section{References}

1 Stember RH: Buckwheat allergy. Allergy Asthma Proc 2006;27:393-395.

2 Park K, Jeong K, Lee S: Clinical and laboratory findings of childhood buckwheat allergy in a single tertiary hospital. Korea J Pediatr 2016;59:402-407.

3 Andersen JB, Kristensen B: Buckwheat allergy can cause live-threatening anaphylaxia. Ugeskr Laeger 2014;176:pii: V08120509.

4 Yanagida N, Sato S, Takahashi K, Nagakura KI, Ogura K, Asaumi T, Ebisawa M: Reactions of buckwheat-hypersensitive patients during oral food challenge are rare, but often anaphylactic. Int Arch Allergy Immunol 2017;172: 116-122.

5 Akiyama H, Imai T, Ebisawa M: Japan food allergen labeling regulation - history and evaluation. Adv Food Nutr Res 2011;62:139171.

6 Takahashi Y, Ichikawa S, Aihara Y, Yokota S: Buckwheat allergy in 90,000 school children in Yokohama. Arerugi 1998;47:26-33.

7 Maruyama N, Sato S, Yanagida N, Cabanos C, Ito K, Borres MP, Moverare R, Tanaka A, Ebisawa $\mathrm{M}$ : Clinical utility of recombinant allergen components in diagnosing buckwheat allergy. J Allergy Clin Immunol Pract 2016;4: e322-e323.

8 Ebisawa M, Ito K, Fujisawa T; Committee for Japanese Pediatric Guideline for Food Allergy; The Japanese Society of Pediatric Allergy and Clinical Immunology: Japanese guidelines for food allergy 2017. Allergol Int 2017; 66:190-204.
9 Muraro A, Werfel T, Hoffmann-Sommergruber K, Roberts G, Beyer K, Bindslev-Jensen C, et al: EAACI food allergy and anaphylaxis guidelines: diagnosis and management of food allergy. Allergy 2014;69:1008-1025.

10 Simons FE, Ardusso LR, Dimov V, Ebisawa M, El-Gamal YM, Lockey RF, Sanchez-Borges M, Senna GE, Sheikh A, Thong BY, Worm M: World Allergy Organization Anaphylaxis Guidelines: 2013 update of the evidence base. Int Arch Allergy Immunol 2013;162:193-204.

11 Yanagida N, Sato S, Asaumi T, Ogura K, Ebisawa M: Risk factors for severe reactions during double-blind placebo-controlled food challenges. Int Arch Allergy Immunol 2017; 172:173-182.

12 Rodriguez-Capote K, Schnabl KL, Maries OR, Janzen P, Higgins TN: Stability of specific IgE antibodies to common food and inhalant allergens. Clin Biochem 2016;49:1387-1389.

13 Henderson CE, Ownby D, Klebanoff M, Levine RJ: Stability of immunoglobulin E (IgE) in stored obstetric sera. J Immunol Methods 1998;213:99-101.

14 Ovary Z, Itaya T, Santomauro J, Richmond G: On the stability of IgE antibodies. J Immunol Methods 1978;24:193-194.

15 Sampson HA: Utility of food-specific IgE concentrations in predicting symptomatic food allergy. J Allergy Clin Immunol 2001; 107:891-896
16 Sato S, Ogura K, Takahashi K, Sato Y, Yanagida N, Ebisawa M: Usefulness of antigen-specific IgE probability curves derived from the 3gAllergy assay in diagnosing egg, cow's milk, and wheat allergies. Allergol Int 2017;66:296301.

17 Komata T, Soderstrom L, Borres MP, Tachimoto H, Ebisawa M: The predictive relationship of food-specific serum IgE concentrations to challenge outcomes for egg and milk varies by patient age. J Allergy Clin Immunol 2007;119:1272-1274.

18 Yanagida N, Minoura T, Takahashi K, Sato S, Ebisawa M: Salmon roe-specific serum IgE predicts oral salmon roe food challenge test results. Pediatr Allergy Immunol 2016;27: 324-327.

19 Borres MP, Maruyama N, Sato S, Ebisawa M: Recent advances in component resolved diagnosis in food allergy. Allergol Int 2016;65: 378-387.

20 Ebisawa M, Moverare R, Sato S, Borres MP, Ito $\mathrm{K}$ : The predictive relationship between peanut- and Ara h 2-specific serum IgE concentrations and peanut allergy. J Allergy Clin Immunol Pract 2015;3:131-132.e131.

21 Ebisawa M, Shibata R, Sato S, Borres MP, Ito $\mathrm{K}$ : Clinical utility of IgE antibodies to $\omega-5$ gliadin in the diagnosis of wheat allergy: a pediatric multicenter challenge study. Int Arch Allergy Immunol 2012;158:71-76. 